\title{
Phase transitions and kinetic properties of gold nanoparticles confined between two-layer graphene nanosheets
}

Gang Wang ${ }^{1}$, Nanhua $\mathrm{Wu}^{1}$, Jionghua Chen ${ }^{4}$, Jinjian Wang ${ }^{1}$, Jingling $\mathrm{Shao}^{1,3}$, Xiaolei $\mathrm{Zhu}^{1 *}$, Xiaohua $\mathrm{Lu}^{1}$, and Lucun $\mathrm{Guo}^{2}$

${ }^{I}$ State Key Laboratory of Materials-Oriented Chemical Engineering, College of Chemical Engineering, Nanjing Tech University, Nanjing 210009, China

${ }^{2}$ College of Materials Science and Engineering, Nanjing Tech University, Nanjing 210009, China

${ }^{3}$ School of Chemistry and Chemical Engineering, Yancheng Institute of technology, Yancheng, 224051, China

${ }^{4}$ Jinling High School, Nanjing, 210005, China

\section{*Corresponding authors}

E-mail addresses: xlzhu@njtech.edu.cn,xhlu@njut.edu.cn 
Abstract The thermodynamic and kinetic behaviors of gold nanoparticles confined between two-layer graphene nanosheets (two-layer-GNSs) are examined and investigated during heating and cooling processes via molecular dynamics (MD) simulation technique. An EAM potential is applied to represent the gold-gold interactions while a Lennard-Jones (L-J) potential is used to describe the gold-GNS interactions. The MD melting temperature of $1345 \mathrm{~K}$ for bulk gold is close to the experimental value (1337K), confirming that the EAM potential used to describe gold-gold interactions is reliable. On the other hand, the melting temperatures of gold clusters supported on graphite bilayer are corrected to the corresponding experimental values by adjusting the $\varepsilon_{\mathrm{Au}-\mathrm{C}}$ value. Therefore, the subsequent results from current work are reliable. The gold nanoparticles confined within two-layer GNSs exhibit face center cubic structures, which is similar to those of free gold clusters and bulk gold. The melting points, heats of fusion, and heat capacities of the confined gold nanoparticles are predicted based on the plots of total energies against temperature. The density distribution perpendicular to GNS suggests that the freezing of confined gold nanoparticles starts from outermost layers. The confined gold clusters exhibit layering phenomenon even in liquid state. The transition of order-disorder in each layer is an essential characteristic in structure for the freezing phase transition of the confined gold clusters. Additionally, some vital kinetic data are obtained in terms of classical nucleation theory.

Keywords Gold nanoparticle, Graphene nanosheet, Phase transition, Molecular dynamics simulation 


\section{Introduction}

The development of the nanometal catalysts with both high stability and high activity is always an important topic of academia and industry[1,2]. In order to realize the sustainable development of catalytic technology, the "bottleneck" problem in the field of catalysis, that is, the contradictory problem of highly active and low stability of the supported nanometal catalysts, is desiderated to be solved[3]. How to raise the stability of supported metal catalysts is very important and challenging.

In recent years, graphene and graphene oxide have drawn much attention because of their novel properties and possible applications[4-6]. Experimentally, graphene and graphene oxide (GO) have been used as supports to improve the stability of metal nanoparticles.[7-12] Although some investigators have successfully synthesized the graphene-supported or graphene oxide-supported gold nanocatalysts[8-12] via different approaches, the structures and physical properties of the confined gold nanoparticles are not clearly understood yet. Molecular dynamics (MD) simulation is powerful technique to solve the above problems. For example, Chien et al.[13] carried out molecular dynamics simulation on gold nanosheet and graphene nanoribbon-gold (GNR/Au) complexes and obtained their stress-strain curves. Lee et al.[14] studied the rotation and diffusion of platinum nanoparticles sustained on two graphene layers. Lee et al.[15] investigated the phase stability of $\mathrm{Pt}_{\mathrm{n}}(\mathrm{n}=38,147,309$ and 561 atoms) nanoclusters with temperature evolution in terms of molecular dynamics (MD) simulation and EAM potential. Results indicate that the different nanoparticles exhibit icosahedral structures within the temperature range of 460-660 K during crystallization. Balbuena et al.[16] studied the shape and structure evolution of platinum nanoclusters on two graphene sheets and observed a temperature-induced wetting 
phenomenon. Jalili et al.[17] applied molecular dynamics simulations investigated the thermal evolution of a cubic copper nanoparticle supported on two graphene sheets. The computed melting temperature of $770 \mathrm{~K}$ is much lower than the bulk melting temperature of copper. Ding et al.[18] studied the structural and dynamic properties of free and supported iron nanoparticles during melting process. They developed an approach for estimating effective diameters of supported metal nanoparticles. In the work of Shibuta et al.[19], molecular dynamics simulation was applied to investigate the liquid-solid phase change of supported molybdenum clusters. The effect of the interaction energy between the substrate and nanocluster on the contact angle, melting, and crystallization temperatures is revealed. Akbarzadeh et al.[20] examined the thermal behavior of graphite-supported $\mathrm{Ag}$ nanoclusters based on molecular dynamics simulations. Results demonstrated that melting temperatures are higher than those of free $\mathrm{Ag}$ nanoclusters with similar size and structure. Additionally, the melting behaviors of some graphite-supported bimetallic nanoparticles were investigated through molecular dynamics simulations [21-24].

Most theoretical studies mentioned above have referred to the structures and dynamic behaviors of the noble metal clusters supported on graphene and graphene oxide. However, there are few reports about the behavior of metal nanoparticles confined between two-layer graphene nanosheets (GNSs) at the atomic level, which will be very difficult for experimental studies. Herein, MD simulation technique is applied to investigate the thermodynamic and kinetic behaviors of the gold nanoparticles confined between two-layer GNSs during melting and freezing processes. Results demonstrate that the gold nanoparticles confined within two-layer GNSs have face center cubic structures. The structural features of the confined gold nanoparticles are revealed during 
freezing processes. Some important kinetic parameters are predicted based on classical nucleation theory. The current work provides some new clues for improving the stability of supported metal catalysts.

\section{Computational details}

We apply MD simulations to investigate the phase change and crystallization of $\mathrm{Au}_{\mathrm{N}}(\mathrm{n}=3990,8778,11970$, and 17157) nanoparticles confined between two-layer GNSs. The length and width of a graphene nanosheet are taken as $234.35 \AA$ and $238.62 \AA$, respectively. Each gold nanoparticle is inserted between two-layer GNSs. The periodic boundary conditions are used along the directions of $\mathrm{x}$ and $\mathrm{z}$ axes. The four

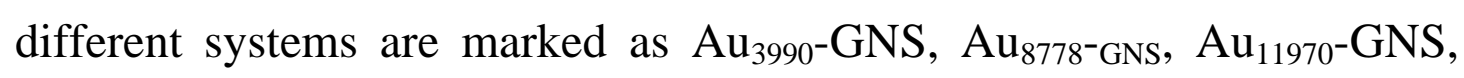
and $\mathrm{Au}_{17157}-\mathrm{GNS}$, respectively.

The EAM potential[25-29] has been confirmed to reproduce both surface properties and bulk of transition metals[30]. In current work, the EAM potential[27] is used to describe the gold-gold interactions, that is

$$
E_{\text {Au-au }}=\sum_{i} F_{i}\left(\rho_{h, i}\right)+\frac{1}{2} \sum_{i} \sum_{j \neq i} \varphi_{i j}\left(r_{i j}\right)
$$

where $F_{\mathrm{i}}\left(\rho_{\mathrm{i}}\right)$ represents the embedding energy of atom $\mathrm{i}, \rho_{h, i}$ displays the host electron density at atom $\mathrm{i}$ formed by the neighboring atoms, $\varphi_{\mathrm{ij}}\left(\mathrm{r}_{\mathrm{ij}}\right)$ accounts for a short-range electrostatic interaction between atoms $i$ and $\mathrm{j}, \mathrm{r}_{\mathrm{ij}}$ illustrates the distance between atoms. In current work, GNS is roughly taken as rigid superstructure with fixed atoms, which is similar to that in previous works[31-33]. On the other hand, a Lennard-Jones (L-J) potential is applied to describe the gold-GNS interactions and relevant L-J parameters are given later .

During the phase transition processes of the systems, first, each confined gold nanocluster is melted above $1600 \mathrm{~K}$. Then, slow cooling run is carried out from $1600 \mathrm{~K}$ to $300 \mathrm{~K}$ with the temperature increments of 
50K (for $1600-1350 \mathrm{~K}$ and $850-300 \mathrm{~K}$ ) and $20 \mathrm{~K}$ (for $880-1300 \mathrm{~K}$ ). The time step is set as $4 \mathrm{fs}$. At each temperature, each system is simulated under a NVT ensemble for $0.4 \mathrm{~ns}$. Subsequently, it is simulated under a NVE ensemble for $0.2 \mathrm{~ns}$. Finally, the slow heating run is performed on each system from $300 \mathrm{~K}$ to $1600 \mathrm{~K}$ with same time step and temperature increment.

To carry out subsequent nucleation analysis, each of the melted clusters $\left(\mathrm{Au}_{3990}, \mathrm{Au}_{8778}\right.$, and $\left.\mathrm{Au}_{11970}\right)$ confined between two-layer GNSs is heated at $1600 \mathrm{~K}$ to get 120 structures of melted clusters with different thermal histories and geometries. Subsequently, the nucleation and crystallization of $\mathrm{Au}_{3990}, \mathrm{Au}_{8778}$, and $\mathrm{Au}_{11970}$ nanoparticles confined inside two-layer GNSs are simulated via quenching the melted $\mathrm{Au}$ nanoparticles to the given temperatures for $0.4 \sim 8 \mathrm{~ns}$.

\section{Results and discussion}

In current work, to examine reliability of the EAM potential of gold, we perform a MD simulation (NPT ensemble) on slow heating process of free bulk gold, the derived MD melting temperature of $1345 \mathrm{~K}$ for bulk gold is close to the experimental value (1337K[34]), confirming that the EAM potential[27] used to describe gold-gold interactions is reliable. On the other hand, the correction of L-J parameters between gold-GNS can be described as follows. We use the gold-carbon L-J potential parameters $\left(\sigma_{\mathrm{Au}-\mathrm{C}}=2.9943 \AA, \varepsilon_{\mathrm{Au}-\mathrm{C}}=0.01273 \mathrm{eV}\right)$ in references[35] and conduct additional MD simulations on several spherical Au clusters with different size supported on a graphite bilayer, the melting points of these $\mathrm{Au}$ clusters are revised to the corresponding experimental data [36] via adjusting the $\varepsilon_{\mathrm{Au}-\mathrm{C}}$ value. When $\varepsilon_{\mathrm{Au}-\mathrm{C}}$ is adjusted as $0.02546 \mathrm{eV}, \mathrm{MD}$ melting points of $\mathrm{Au}$ clusters satisfy with the experimental results very 
well.[36] Thus, in this work, gold-carbon L-J potential parameters are taken as $\sigma_{\mathrm{Au}-\mathrm{C}}=2.9943 \AA, \varepsilon_{\mathrm{Au}-\mathrm{C}}=0.02546 \mathrm{eV}$. The above analysis for gold-gold interactions and correction for gold-GNS interactions suggest that the subsequent results from our work are credible.

Figure S1(see Supplementary Material) illustrates the plot of total energy against temperature for $\mathrm{Au}_{3990}, \mathrm{Au}_{8778}, \mathrm{Au}_{11970}$ and $\mathrm{Au}_{17157}$ confined between two-layer GNSs during heating processes. The melting temperatures of the confined gold nanoparticles $\left(\mathrm{Au}_{3990}, \mathrm{Au}_{8778}, \mathrm{Au}_{11970}\right.$, and $\left.\mathrm{Au}_{17157}\right)$ are $1148,1075,1064$, and $1050 \mathrm{~K}$, respectively, as represented in Fig. S1, which implies that the melting temperatures of confined Au nanoparticles are inclined to decrease with nanoparticle size and will be interpreted latter. For the above four systems $\left(\mathrm{Au}_{\mathrm{N}}-\mathrm{GNS}, \mathrm{N}=\right.$ 3990, 8778, 11970, and 17157), the melting temperatures, heat capacities, and heats of fusion listed in Table 1 are derived from heating curves of Fig. S1. In order examine the confined strength of GNS substrate on the melting temperatures of $\mathrm{Au}$ clusters, during the additional MD simulations for heating processes, potential well depth values $\left(\varepsilon_{\mathrm{Au}-\mathrm{C}}\right)$ between gold cluster and support are set as $0.35 \varepsilon_{\mathrm{Au}-\mathrm{C}}, 0.5 \varepsilon_{\mathrm{Au}-\mathrm{C}}$, and $1.5 \varepsilon_{\mathrm{Au}-\mathrm{C}}$, respectively, in which the support (or carrier) is changed. As shown in Fig. 1, the melting temperatures increase with interaction strength between gold and support, suggesting that the stronger interaction between gold and support can stabilize gold nanoparticles. Additionally, Fig. S2 represents the temperature dependence of total energy for $\mathrm{Au}_{8778}$-GNS during cooling and heating processes, which suggests that during cooling process, there exists a hysteresis phenomenon [19] for freezing of the confined gold nanoparticle.

The structures of the confined $\mathrm{Au}_{\mathrm{N}}(\mathrm{N}=3990,8778$, and 11970) clusters at $300 \mathrm{~K}$ are displayed in Fig. S3 (see Supplementary Material). 
Clearly, as shown in Fig. S3, the confined gold nanoparticles have face center cubic (FCC) structures, which is similar to those of bulk $\mathrm{Au}$ and free Au nanoparticles [37]. Interestingly, Fig. S3(b) demonstrates that for each confined solid gold nanoparticle, the crystal plane parallel to GNS exhibits quasi-hexagon structure. In fact, there does exist a good epitaxy between the hexagonal carbon network of graphene and the closed-packed facet of metals. The preliminary results in Fig. S4 and Fig. S5 demonstrate the structure of the graphene and outermost layer of gold nanoparticle for confined $\mathrm{Au}_{3990}$ nanoparticle. Such a good epitaxy enhances the heterogeneous nucleation, which may be called the complementary heterogeneous nucleation [38]. The investigation about epitaxy mechanism in details between the graphene and outermost layer of gold nanoparticles is in the progress.

Figure 2 illustrates the images of the atoms in the confined $\mathrm{Au}_{8778}$ nanoparticle during cooling and heating processes, which can be applied to examine the melting transition. Clearly, after freezing or before melting, $\mathrm{Au}_{8778}$ clusters have ordered FCC structures (Figs. 2 (c), (d), and (e)), and before freezing or after melting, $\mathrm{Au}_{8778}$ exhibit disordered structures (Figs. 2 (a),(b),(f), and (g)). As shown in Fig. 3, the density distribution of the confined $\mathrm{Au}_{8778}$ along the direction perpendicular to GNS is used to examine and reveal the characteristic of freezing for the confined $\mathrm{Au}$ clusters. After freezing, the twenty-two peaks can be observed on the density curve, implying that the $\mathrm{Au}_{8778}$ exhibits 22-layer structure prior to melting (at $300 \mathrm{~K}$ and $700 \mathrm{~K}$ in Fig. 3). It is worth noting from Fig. 3 that the outermost layers form first during cooling process, revealing that nucleation and crystallization start from the outermost layer of confined $\mathrm{Au}_{8778}$, which are similar to those of the gold nanoparticles within SWNTs[39]. On the other hand, before freezing, a confined gold cluster 
exhibits layering behavior, which implies the layering phenomenon is not essential feature of freezing for the confined gold nanoparticle. Therefore, we further examine the structure of each layer for a confined gold cluster before and after freezing. Figure 4 indicates the structures of surface atoms of $\mathrm{Au}_{8778}$ within two-layer GNSs at 1100 and $1000 \mathrm{~K}$, respectively. Obviously, the atom arrangement of outermost layer emerges disordered conformation prior to freezing (Fig. 4 (a)(1100K). However, they become the ordered triangle structures in crystalline $\mathrm{Au}_{8778}$ (Fig. 4 (b) (1000K)). Their interior layers also appear to be similar structures, revealing that transition of order-disorder in each layer is an intrinsical characteristic for the freezing phase transition of a confined gold nanoparticle.

As mentioned above, the melting points of the confined gold nanoparticles $\left(\mathrm{Au}_{3990}, \mathrm{Au}_{8778}, \mathrm{Au}_{11970}\right.$, and $\left.\mathrm{Au}_{17157}\right)$ are 1148, 1075, 1064, and $1050 \mathrm{~K}$, respectively, which demonstrate an abnormal behavior of the gold nanoparticles confined between the two-layer GNSs, that is, the melting temperatures of confined Au nanoparticles tend to decrease with nanoparticle size. It is can be explained as follows. First, the melting points of the confined gold nanoparticles are higher than those of free gold nanoparticles as expected. Second, Fig.3 implies that the melting the confined gold nanoparticle starts from the inner layer while the free nanoparticles melt starts from their surfaces. For the confined $\mathrm{Au}_{8778}$ nanoparticle between two-layer GNSs, the interactions of GNSs on the surface atoms of $\mathrm{Au}_{8778}$ nanoparticle are stronger than those of GNSs on inner gold atoms. As a result, the melting temperature of centermost layer is lower than that of surface layers for confined $\mathrm{Au}_{8778}$. In other word, for the confined gold nanoparticles, the centermost-layer atoms are distant from the two-layer GNSs as gold nanorods increases in length, the confined strength of GNSs on Au clusters gradually weaken. Therefore, the melting points of confined gold nanoparticles are gradually reduced to 
those of free nanoparticles with same size. Third, for the confined gold nanoparticles, the increasing of melting temperature caused by confined environment of two-layer-GNS outstrips the decreasing of melting temperature due to decreasing of particle size. Thus, the melting temperatures of confined $\mathrm{Au}$ nanoparticles tend to decrease with their size.

Subsequently, in order to explore nucleation process and predict dynamic parameters for the confined gold nanoparticles, we apply the classical nucleation theory to perform the nucleation analysis. So far, the solid-liquid interfacial free energy $\sigma_{s l}$ of confined gold is not easy to get from experiment. Fortunately, the Turnbull relation[40] and simulated results can be used to estimate the interfacial free energy $\left(\sigma_{s l}\right)$ via the following relationship,

$$
\sigma_{s l}=k_{T} \Delta H_{\text {fus }} /\left(V^{2 / 3} N_{A}^{1 / 3}\right)
$$

where the $\mathrm{k}_{\mathrm{T}}$ is the constant of 0.45 [40]. The $\mathrm{V}$ illustrates molar volume and $\Delta H_{\text {fus }}$ represents fusion heat. Based on eq. (2) and $\Delta H_{\text {fus }}$ values listed in Table 1, the computed $\sigma_{s l}$ values for the confined gold nanoparticles are $0.095 \sim 0.106 \mathrm{~J} / \mathrm{m}^{2}$ as illustrated in Table 2.

The nucleation rate $(\mathrm{J})$ is generally represented by[41]

$\ln J=\ln A-\Delta G^{*} /\left(k_{B} T\right)$

where $\Delta \mathrm{G}^{*}$ indicates the nucleation energy barrier for formation of a critical nucleus from the melt and the prefactor A can be computed via classical nucleation theory, that is,

$A=16(3 / 4 \pi)^{1 / 3} \sigma_{s I}^{1 / 3} D /\left(k_{B} T V_{m}^{4 / 3}\right)$

where $V_{m}$ represents the molecular volume in crystal. The coefficients of self-diffusion (D) in melted gold clusters can be calculated in terms of the slow cooling processes and the following relation, 
$D=\left(d\left\langle r^{2}(t)\right\rangle / d t\right) / 6$

Fig. S6 (see Supplementary Material) displays the plot of diffusion coefficients against temperature. Based on Fig. S6 and the empirical Arrhenius equation, the activation energies and prefactors can be derived, which can be used to analyze dynamic parameters.

During nucleation simulation processes, the nucleation rates $(\mathrm{J})$ can be obtained via conducting 30 independent quenching runs on the confined gold clusters in melted state for each cluster size and each freezing temperature. It is supposed that at a given temperature, the fraction of unfrozen nanoparticles $\left(N_{n}\right)$ abides by the first-order rate law and can be expressed by,

$N_{n} / N_{0}=\exp \left[-J V_{c}\left(t_{n}-t_{0}\right)\right]$

In the eq (6), $t_{n}$ and $t_{n}$ account for nucleation time and time lag, respectively, $\mathrm{N}_{0}$ is the number of nanoparticles, and $\mathrm{V}_{\mathrm{c}}$ can be roughly set as the volume of gold nanoparticle. Using the curve of total energy with time[42] (Fig. S7 (see Supplementary Material), the nucleation times can be estimated. Each set of data in Fig. $5\left(\ln \left(\mathrm{N}_{\mathrm{n}} / \mathrm{N}_{0}\right) \sim\right.$ time $)$ is linearly fitted. Obviously, the nucleation rates ( $\mathrm{J}$ ) can be derived in terms of the slopes of the fitting lines of data in Fig. 5. It is noted from Table 2 and Fig. 6 that the nucleation rates appears to increase with the decreasing of cluster size. Because the length of gold nanorod is approximately equal to the distance between two GNSs (called as hole diameter of two-layer GNSs hereunder), the above result implies that the nuclei are smaller in two-layer-GNS support with smaller hole diameter. On the other hand, it is noted from Fig. S1 that the melting temperatures are higher for the small gold clusters confined two-layer-GNS support with smaller hole diameter, which reveals that the two-layer-GNS support with smaller hole diameter can stabilize smaller gold nanoparticles. 
As mentioned above, the results in Fig. 3 demonstrate that the nucleation initially occurs at the interface between Au cluster and GNS. Obviously, when the distance between two GNSs is shorter, the surface-to-volume ratio of Au cluster is larger, which provides more sites where nuclei can be formed. On the other hand, because the increasing of the nucleation barrier energy with temperature dominates nuclei formation, the nucleation rates are inclined to decrease with temperature as shown in Fig. 6. The obtained nucleation rates of confined Au clusters are about $10^{34} \sim 10^{35} \mathrm{~m}^{-1} \mathrm{~s}^{-1}$ as shown in Table 2 and Fig. 6, which are smaller than those of the gold nanoparticles confined within SWNT[39] due to the different confined environment. Additionally, the nucleation energy barriers $\left(\Delta G^{*}\right)$ can be predicted to be $1 \sim 2 \times 10^{-19} \mathrm{~J}$ in terms of eqs (2) (6) as illustrated in Fig. 7. It is easy to note from Fig. 7 that the nucleation barrier increases with temperature as expected. Interestingly and importantly, it can be noted from Fig. 7 that if the pore diameter of two-layer-GNS support is smaller, the nucleation barrier is smaller and the nucleation rate is larger, which is identical with the results of Fig. 6.

\section{Conclusions}

The melting and crystallization behaviors of gold nanoparticles confined between two-layer GNSs are investigated and explored via MD simulation technique. Several important results can be described as follows. (i) The confined gold nanoparticles have face center cubic structures, which is similar to those of bulk Au and free Au nanoparticles; (ii) The melting temperatures of the confined gold nanoparticles are estimated based on the plots of total energy against temperature; (iii) The results of density distribution perpendicular to GNS reveal that the confined gold nanoparticles freeze start from the outmost layers. The 
confined gold clusters exhibit layering behavior even in liquid state. The transition of order-disorder in each layer is an entitative structural characteristic for the freezing transition of a confined gold cluster; (iv) the two-layer-GNS support with smaller hole diameter can stabilize smaller gold nanoparticles; (v) The interfacial free energies predicted by Turnbull relation are $0.095 \sim 0.106 \mathrm{~J} / \mathrm{m}^{2}$. The nucleation rates are inclined to increase with the decreasing of cluster size, implying the nuclei are smaller in smaller hole diameter of two-layer-GNS support. The nucleation rates tend to decrease with temperature. The obtained nucleation rates of confined Au clusters are about $10^{34} \sim 10^{35} \mathrm{~m}^{-1} \mathrm{~s}^{-1}$, which are smaller than those of the gold nanoparticles confined within SWNT due to the different confined environment. The nucleation energy barrier $\left(\Delta G^{*}\right)$ values are predicted to be $1 \sim 2 \times 10^{-19} \mathrm{~J}$. The results in current work provide a guide and basic data for improving the stability of supported metal catalysts.

\section{Acknowledgment}

This work is supported by grants from the National Science Foundation of China (Nos. 21276122, 21136001), the Major Research Plan of the National Natural Science Foundation of China (Nos. 91334202, 91434109), and State Key Laboratory of Materials-Oriented Chemical Engineering, College of Chemistry and Chemical Engineering, Nanjing Tech University of China (No. ZK201212). We want to express our thanks for Reviewers' valuable suggestions for this article.

\section{Appendix A. Supplementary Material}


Supplementary material Fig. S1: Temperature dependence of total energy for $\mathrm{Au}_{\mathrm{N}}(\mathrm{N}=3990,8778,11970$, and 17157) nanoparticles confined between two-layer GNSs during heating process. Fig. S2: Temperature dependence of total energy for $\mathrm{Au}_{8778}$-GNS during heating and cooling processes. Fig. S3: Images of the confined $\mathrm{Au}_{\mathrm{N}}(\mathrm{N}=3990,8778,11970$, and 17157) at 300K. (a) and (b) for $\mathrm{Au}_{3990}-\mathrm{GNS}$; (c) for $\mathrm{Au}_{8778}$-GNS; (d) for $\mathrm{Au}_{11970}$-GNS. Fig. S4: The structure of the graphene and outermost layer of gold nanoparticle for confined $\mathrm{Au}_{3990}$ nanoparticle. Fig. S5: The structure of crystalline $\mathrm{Au} 3990$ nanoparticle on graphene nanosheet (green). Fig. S6: The relationship between the diffusion coefficient and temperature. Fig. S7 : Time dependences of the total energy for $\mathrm{Au}_{8778}$-GNS.

\section{References}

[1] L. Liang, J.L. Shi, Synthesis of rhodium colloidal nano-coating grafted mesoporous silica composite and its application as efficient environmentally benign catalyst for heck-type reaction of arylboronic acids, Adv. Synth. Catal. 350 (2008) 667-672.

[2] R. Redon, N.G. Garcia-Pena, V.M. Ugalde-Saldivar, J.J. Garcíab, Palladium-triazine aminoalcohol nanocomposite, its reactivity on heck reaction, J. Mol. Catal. A-Chem. 300 (2009) 132-141.

[3] P. Euzen, J.H. Le Gal, B. Rebours, Deactivation of palladium catalyst in catalytic combustion of methane, Catal. Today 47 (1999) 
[4] A.K. Geim, Graphene: Status and Prospects, Science 324 (2009) 1530-1534.

[5] A.B. Bourlinos, D. Gournis, D. Petridis, T. Szabo, A. Szeri, I. Dekany, Graphite oxide: chemical reduction to graphite and surface modification with primary aliphatic amines and aminoacids, Langmuir 19 (2003) 6050-6055.

[6] Y.B. Zhang, Y.W. Tan, H.L. Stormer, P. Kim, Experimental observation of the quantum hall effect and berry's phase in graphene, Nature 438 (2005) 201-204.

[7] Y.C. Si, E.T. Samulski, Exfoliated graphene separated by platinum nanoparticles, Chem. Mater. 20 (2008) 6792-6797.

[8] X. Liu, X. Wang, P. He, L. Yi, Z. Liu, X. Yi, Influence of borohydride concentration on the synthesized Au/graphene nanocomposites for direct borohydride fuel cell, J. Solid State Electr. 16 (2012) 3929-3937.

[9] X. Dang, X. Zhang, Z. Lu, Z. Yang, X. Dong, X. Zhang, C. Ma, H. Ma, M. Xue, F. Shi, Construction of $\mathrm{Au} @ \mathrm{TiO}_{2} /$ graphene nanocomposites with plasmonic effect and super adsorption ability for enhanced visible-light-driven photocatalytic organic pollutant degradation, J. Nanopart. Res. 16 (2014) 2215.

[10] J. Huang, L. Zhang, B. Chen, N. Ji, F. Chen, Y. Zhang, Z. Zhang, Nanocomposites of size-controlled gold nanoparticles and graphene oxide: formation and applications in SERS and catalysis, Nanoscale 2 (2010) 2733-2738.

[11] T. Wu, J. Ma, X. Wang, Y. Liu, H. Xu, J. Gao, W. Wang, Y. Liu, J. Yan, Graphene oxide supported $\mathrm{Au}-\mathrm{Ag}$ alloy nanoparticles with different shapes and their high catalytic activities, Nanotechnology 24 (2013) 125301. 
[12] J. Chen, G. Cheng, Z. Li, F. Miao, X. Cui, W. Zheng, Ultrafine au nanodots on graphene oxide for catalytic reduction of 4-Nitrophenol, Nano 08 (2013) 1350034.

[13] S.K. Chien, Y.T. Yang, C.K. Chen, A molecular dynamics study of the mechanical properties of graphene nanoribbon-embedded gold composites, Nanoscale 3 (2011) 4307-4313.

[14] J.H. Ryu, D.H. Seo, H. Kim da, H.M. Lee, Molecular dynamics simulations of the diffusion and rotation of Pt nanoclusters supported on graphite, Phys. Chem. Chem. Phys. 11 (2009) 503-507.

[15] S.H. Lee, S.S. Han, J.K. Kang, J.H. Ryu, H.M. Lee, Phase stability of Pt nanoclusters and the effect of a $\left(\begin{array}{llll}0 & 0 & 0 & 1\end{array}\right)$ graphite surface through molecular dynamics simulation, Surf. Sci. 602 (2008) 1433-1439.

[16] S.P. Huang, P. Balbuena, Platinum nanoclusters on graphite substrates: a molecular dynamics study, Mol. Phys. 100 (2002) 2165-2174.

[17] S. Jalili, C. Mochani, M. Akhavan, J. Schofield, Molecular dynamics simulation of a graphite-supported copper nanocluster: Thermodynamic properties and gas adsorption, Mol. Phys. 110 (2012) 267-276.

[18] F. Ding, A. Rosén, S. Curtarolo, K. Bolton, Modeling the melting of supported clusters, Appl. Phys. Lett. 88 (2006) 133110-133112.

[19] Y. Shibuta, T. Suzuki, Phase transition in substrate-supported molybdenum nanoparticles: a molecular dynamics study, Phys. Chem. Chem. Phys. 12 (2010) 731-739.

[20] H. Akbarzadeh, H. Yaghoubi, S. Salemi, Investigation of thermal behavior of graphite-supported Ag nanoclusters of different sizes using molecular dynamics simulations, Fluid Phase Equilibr. 365 (2014) 68-73. [21] F.N. Carlos, M.R. Sergio, Molecular dynamics of free and graphite-supported Pt-Pd nanoparticles, Advances in Nanoparticles 2 
(2013) 323-328.

[22] S.K.R.S. Sankaranarayanan, V.R. Bhethanabotla, B. Joseph, Molecular dynamics simulations of the structural and dynamic properties of graphite-supported bimetallic transition metal clusters, Phys. Rev. B 72 (2005) 195405.

[23] S.P. Huang, D.S. Mainardi, P.B. Balbuena, Structure and dynamics of graphite-supported bimetallic nanoclusters, Surf. Sci. 545 (2003) 163-179.

[24] B.H. Morrow, A. Striolo, Supported bimetallic Pt-Au nanoparticles: Structural features predicted by molecular dynamics simulations, Phys. Rev. B 81 (2010) 155437.

[25] M.S. Daw, M.I. Baskes, Embedded-atom method: Derivation and application to impurities, surfaces, and other defects in metals, Phys. Rev. B 29 (1984) 6443-6453.

[26] S.M. Foiles, M.I. Baskes, M.S. Daw, Embedded-atom-method functions for the fcc metals $\mathrm{Cu}, \mathrm{Ag}, \mathrm{Au}, \mathrm{Ni}, \mathrm{Pd}, \mathrm{Pt}$, and their alloys, Phys. Rev. B 33 (1986) 7983-7991.

[27] J.B. Adams, S.M. Foiles, W.G. Wolfer, Self-diffusion and impurity diffusion of FCC metals using the 5-frequency model and the embedded atom method, J. Mater. Res. 4 (1989) 102-112.

[28] S.S. Hayat, M.A. Choudhry, S.A. Ahmad, J.I. Akhter, Hussain, Study of thermal properties of nickel using embedded-atom-method, A. Indian. J. Pure Ap. Phy. 46 (2008) 771-775.

[29] J. Mei, J.W. Davenport, Molecular dynamics study of self-diffusion in liquid transition metals, Phys. Rev. B 42 (1990) 9682-9684.

[30] M.S. Daw, S.M. Foiles, M.I. Baskes, The embedded-atom method: a review of theory and applications, Mater. Sci. Rep. 9 (1993) 251-310.

[31] S. Arcidiacono, J.H. Walther, D. Poulikakos, D. Passerone, P. 
Koumoutsakos, Solidification of gold nanoparticles in carbon nanotubes, Phys. Rev. Lett. 94 (2005) 105502.

[32] H.J. Hwang, O.K. Kwon, J.W. Kang, Copper nanocluster diffusion in carbon nanotube, Solid State Commun. 129 (2004) 687-690.

[33] J.W. Kang, H.J. Hwang, Structural properties of caesium encapsulated in carbon nanotubes, Nanotechnology 15 (2004) 115-119.

[34] Weast, R. C. CRC handbook of Chemistry and Physics, 63rd ed., CRC Press: Boca Raton, FL, 1982.

[35] W. D. Luedtke, U. Landman, Phys. Rev. Lett. 82 (1999), 3835.

[36] P. Buffat, J. P. Borel, Phys. Rev. B 13(1975), 2287.

[37] Y.G. Chushak, L.S. Bartell, Melting and freezing of gold nanoclusters, J. Phys. Chem. B 105 (2001) 11605-11614.

[38] J. A. Elliott, Y. Shibuta, H. Amara, C. Bichara and E. C. Neyts, Atomistic modelling of CVD synthesis of carbon nanotubes and graphene, Nanoscale 5 (2013) 6662-6676.

[39] J.L. Shao, C. Yang, X.L. Zhu, X.H. Lu, Melting and freezing of Au nanoparticles confined in armchair single-walled carbon nanotubes, $\mathrm{J}$. Phys. Chem. C 114 (2010) 2896-2902.

[40] D. Turnbull, Formation of crystal nuclei in liquid metals, J. Appl. Phys. 21 (1950) 1022-1028.

[41] L.S. Bartell, J.F. Huang, Molecular dynamics investigation of the nucleation barrier and prefactor in the freezing of $(\mathrm{NaCl})_{108}$ clusters, $\mathrm{J}$. Phys. Chem. A 102 (1998) 8722-8726.

[42] J.F. Huang, X.L. Zhu, L.S. Bartell, Molecular dynamics studies of the kinetics of freezing of $(\mathrm{NaCl})_{108}$ clusters, J. Phys. Chem. A 102 (1998) 2708-2715. 


\section{Figure captions}

Fig. 1. The curve of total energy with temperature for the confined $\mathrm{Au}_{8778}$ with different $\mathrm{Au}-\mathrm{GNS}$ interactions ( $\varepsilon$ values are set as $0.35 \varepsilon_{\mathrm{Au}-\mathrm{C}}$, $0.5 \varepsilon_{\mathrm{Au}-\mathrm{C}}, 1 \varepsilon_{\mathrm{Au}-\mathrm{C}}$, and $1.5 \varepsilon_{\mathrm{Au}-\mathrm{C}}$, respectively).

Fig. 2. Images of $\mathrm{Au}_{8778}$ confined between two-layer GNSs at different cooling and heating stages. Cooling process (top): (a)1400K; (b)1100K; (c)700K; (d) 300K; heating process (bottom):(e)700K; (f)1100K; (g)1400K. The pink and blue balls account for gold and carbon atoms, respectively.

Fig. 3. Density distribution along the direction of perpendicular to GNS

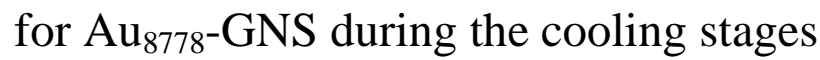

Fig. 4. The structure of outermost layer for $\mathrm{Au}_{8778}$ confined between two-layer GNSs perpendicular to GNS at different temperatures.

Fig. 5. Plots of $\ln \left(\mathrm{N}_{\mathrm{n}} / \mathrm{N}_{0}\right)$ against nucleation time.(a) $\mathrm{Au}_{3990}-\mathrm{GNS}$; (b)

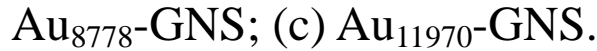

Fig. 6. The relationship between temperature and nucleation rate for the confined $\mathrm{Au}_{\mathrm{N}}(\mathrm{N}=3990,8778$, and 11970) clusters. 
Fig. 7. The correlation of nucleation barrier with temperature for $\mathrm{Au}_{3990},-\mathrm{GNS}, \mathrm{Au}_{8778^{-}} \mathrm{GNS}$, and $\mathrm{Au}_{11970^{-}} \mathrm{GNS}$. 


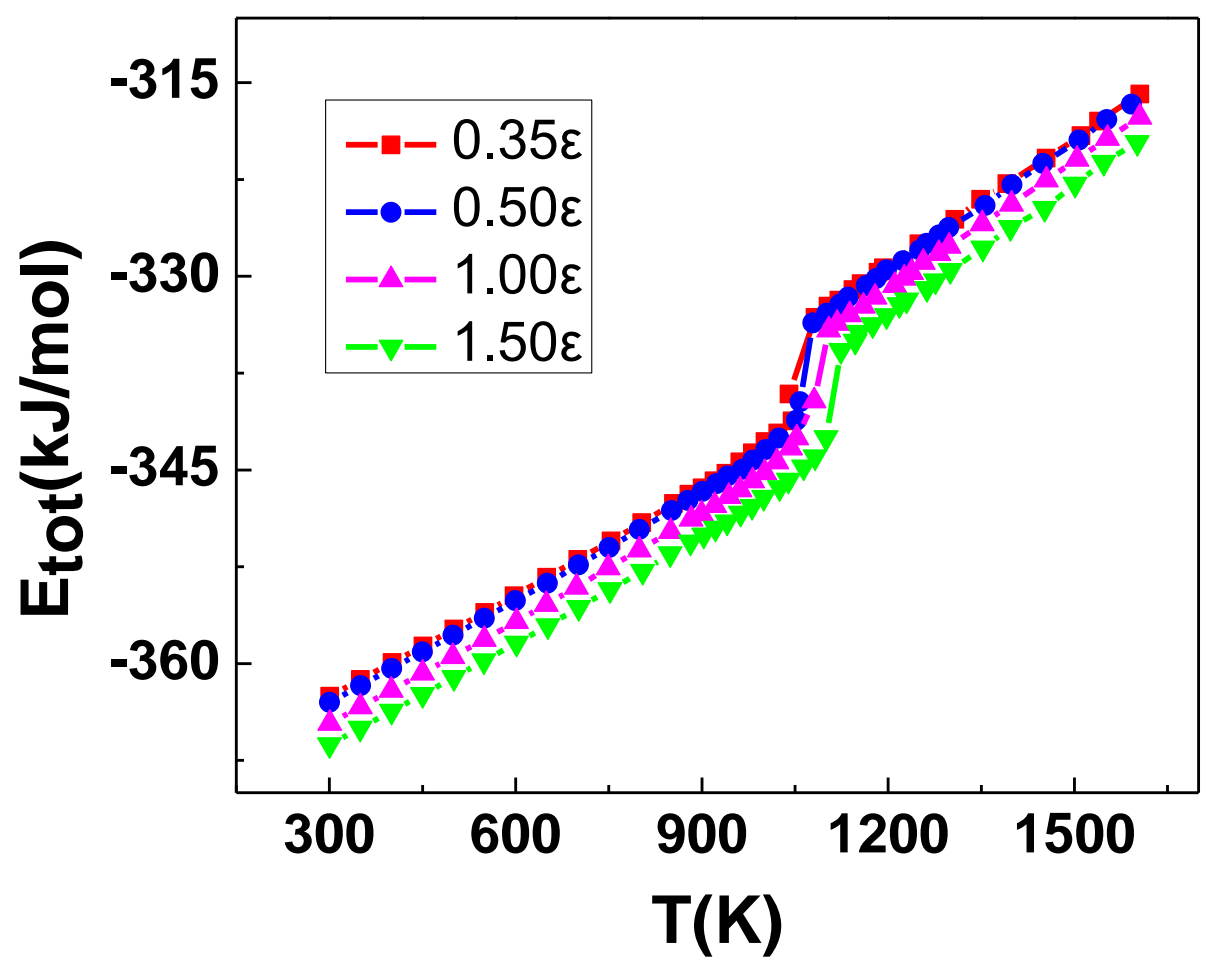

Fig. 1. The plot of total energy against temperature for the confined $\mathrm{Au}_{8778}$ with different $\mathrm{Au}-\mathrm{GNS}$ interactions ( $\varepsilon$ values are set as $0.35 \varepsilon_{\mathrm{Au}-\mathrm{C}}$, $0.5 \varepsilon_{\mathrm{Au}-\mathrm{C}}, 1 \varepsilon_{\mathrm{Au}-\mathrm{C}}$, and $1.5 \varepsilon_{\mathrm{Au}-\mathrm{C}}$, respectively). 


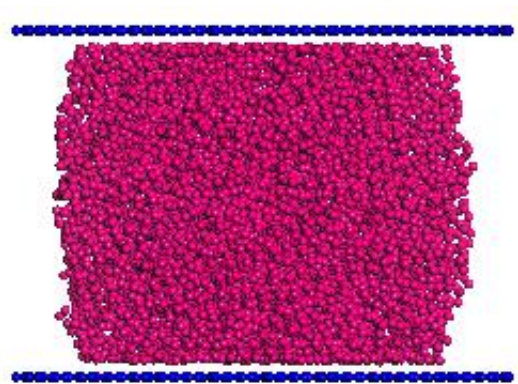

(a) $1400 \mathrm{~K}$

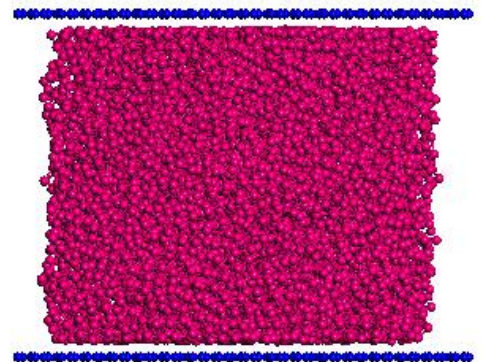

(b) $1100 \mathrm{~K}$

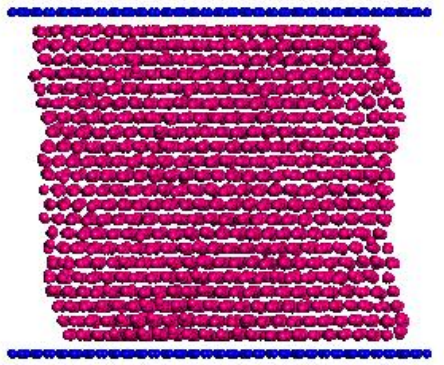

(c) $700 \mathrm{~K}$

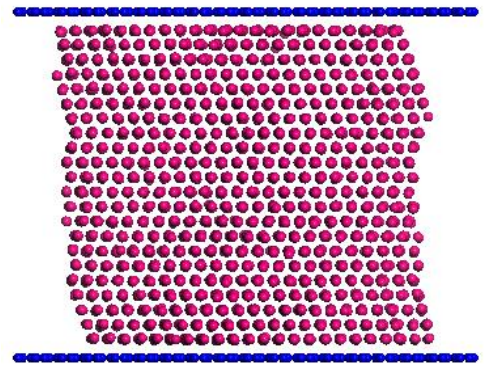

(d) $300 \mathrm{~K}$

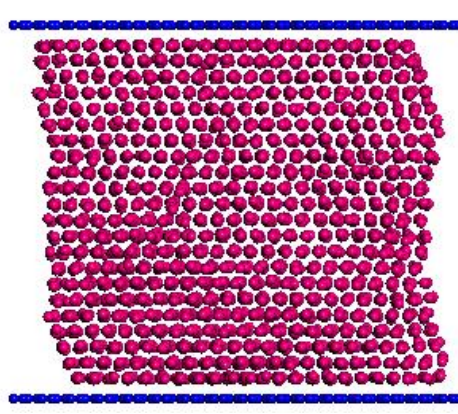

(e) $700 \mathrm{~K}$

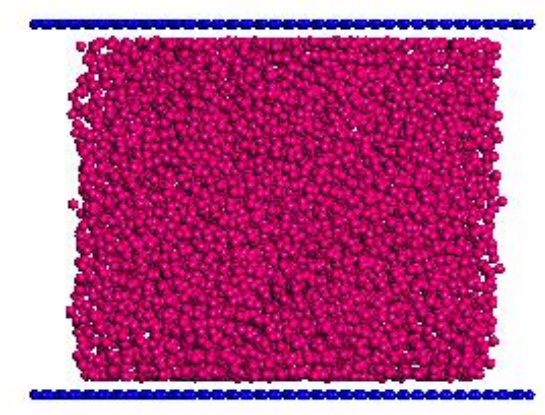

(f) $1100 \mathrm{~K}$

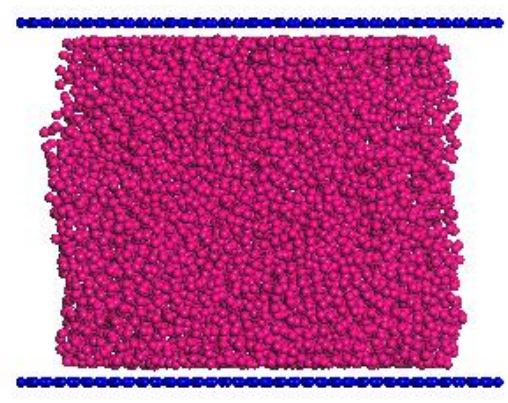

(g) $1400 \mathrm{~K}$

Fig. 2. Images of $\mathrm{Au}_{8778}$ confined between two-layer GNSs at various stages of cooling and heating. Cooling stages (top): (a)1400K; (b)1100K; (c)700K; (d) 300K; heating stages (bottom):(e)700K; (f)1100K; (g)1400K. The pink and blue balls account for gold and carbon atoms, respectively. 


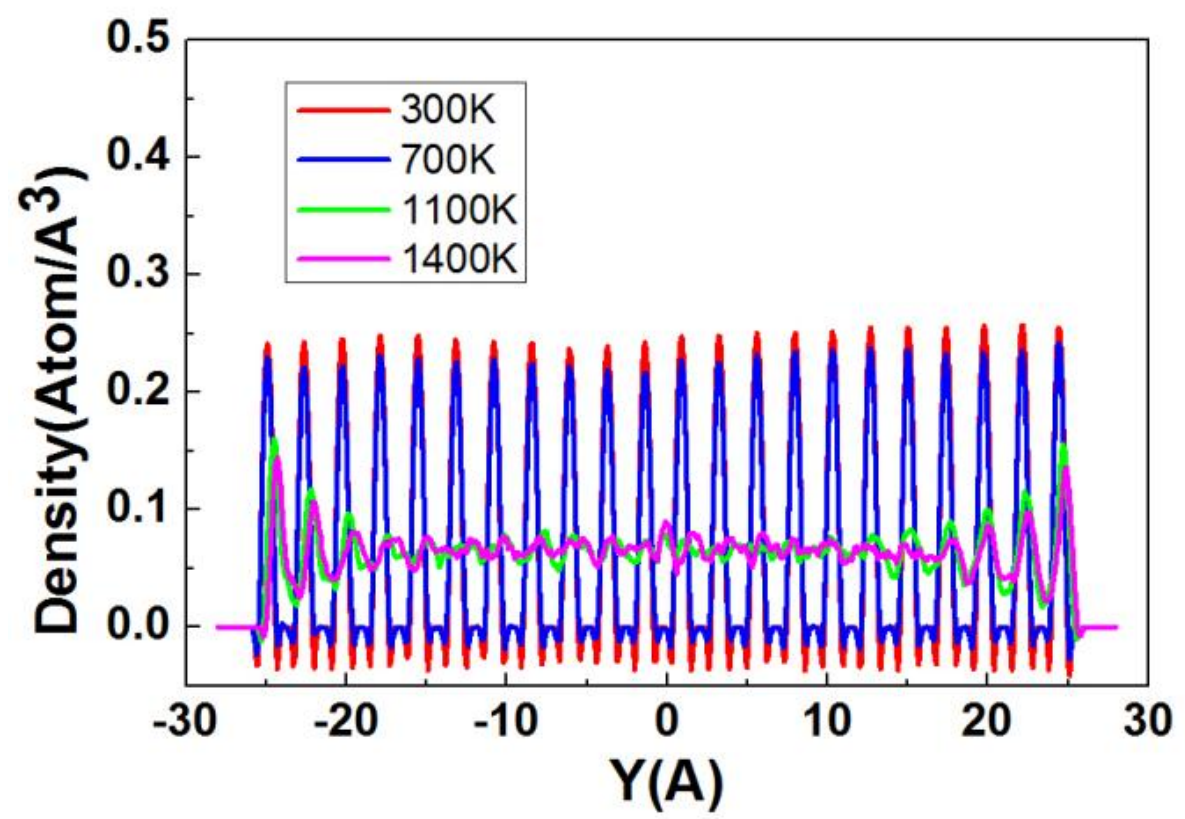

Fig. 3. Density distribution along the direction of perpendicular to GNS for $\mathrm{Au}_{8778}$-GNS during the cooling stages 


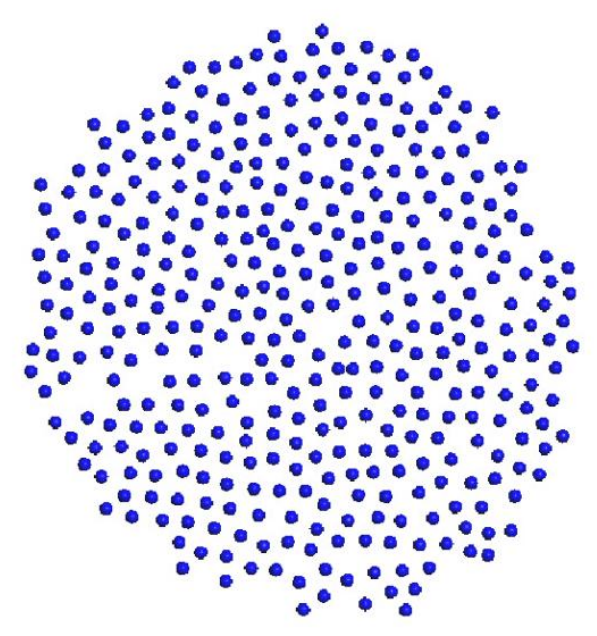

(a) $1100 \mathrm{~K}$

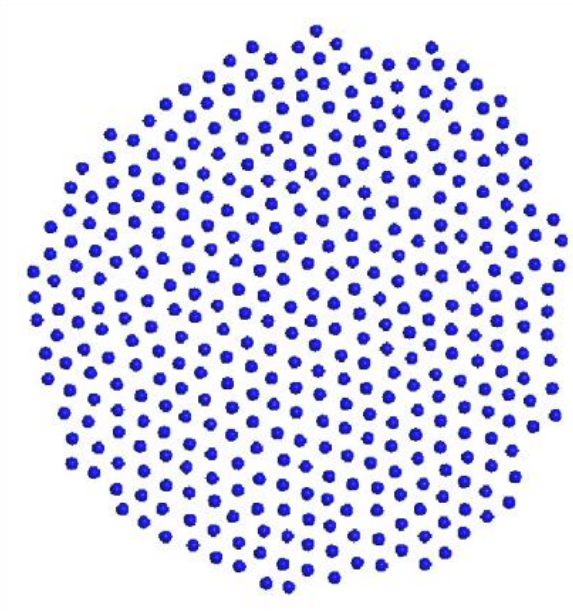

(b) $1000 \mathrm{~K}$

Fig. 4. The structure of outermost layer for $\mathrm{Au}_{8778}$ confined between two-layer GNSs perpendicular to GNS at different temperatures. 

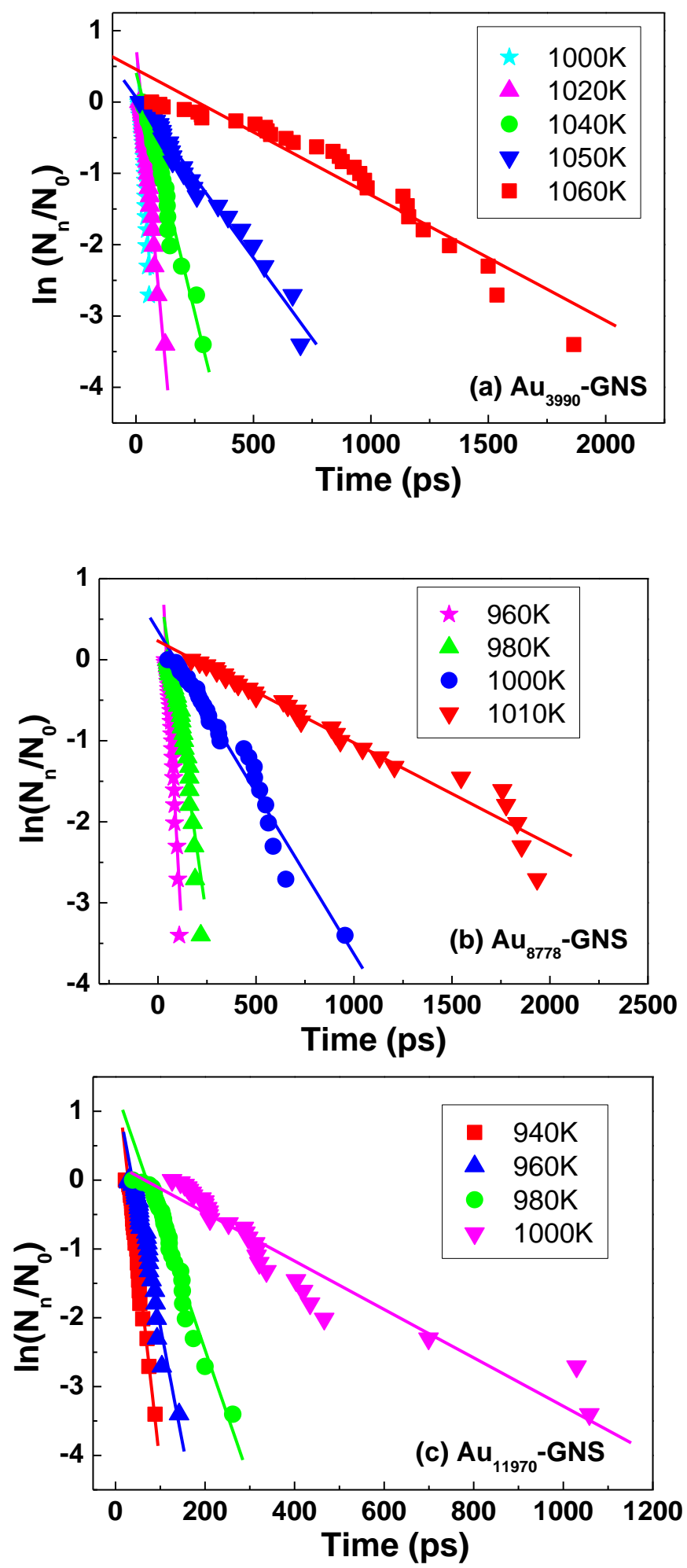

Fig. 5. Plots of $\ln \left(\mathrm{N}_{\mathrm{n}} / \mathrm{N}_{0}\right)$ against nucleation time.(a) $\mathrm{Au}_{3990}$-GNS; (b) $\mathrm{Au}_{8778}-\mathrm{GNS} ;$ (c) $\mathrm{Au}_{11970-\mathrm{GNS}}$ 


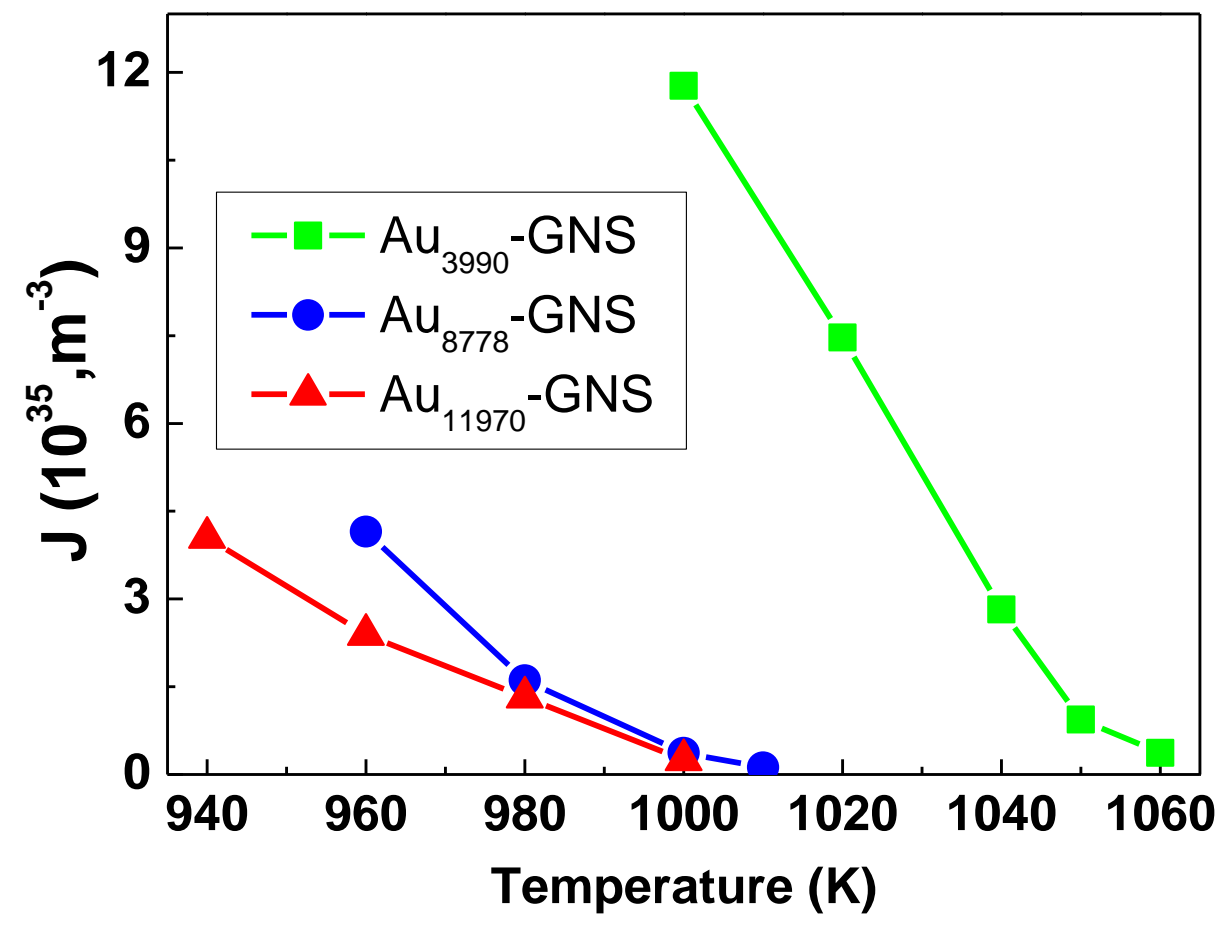

Fig. 6. Temperature dependence of nucleation rate for the confined $\mathrm{Au}_{\mathrm{N}}$ ( $N=3990,8778$, and 11970) clusters. 


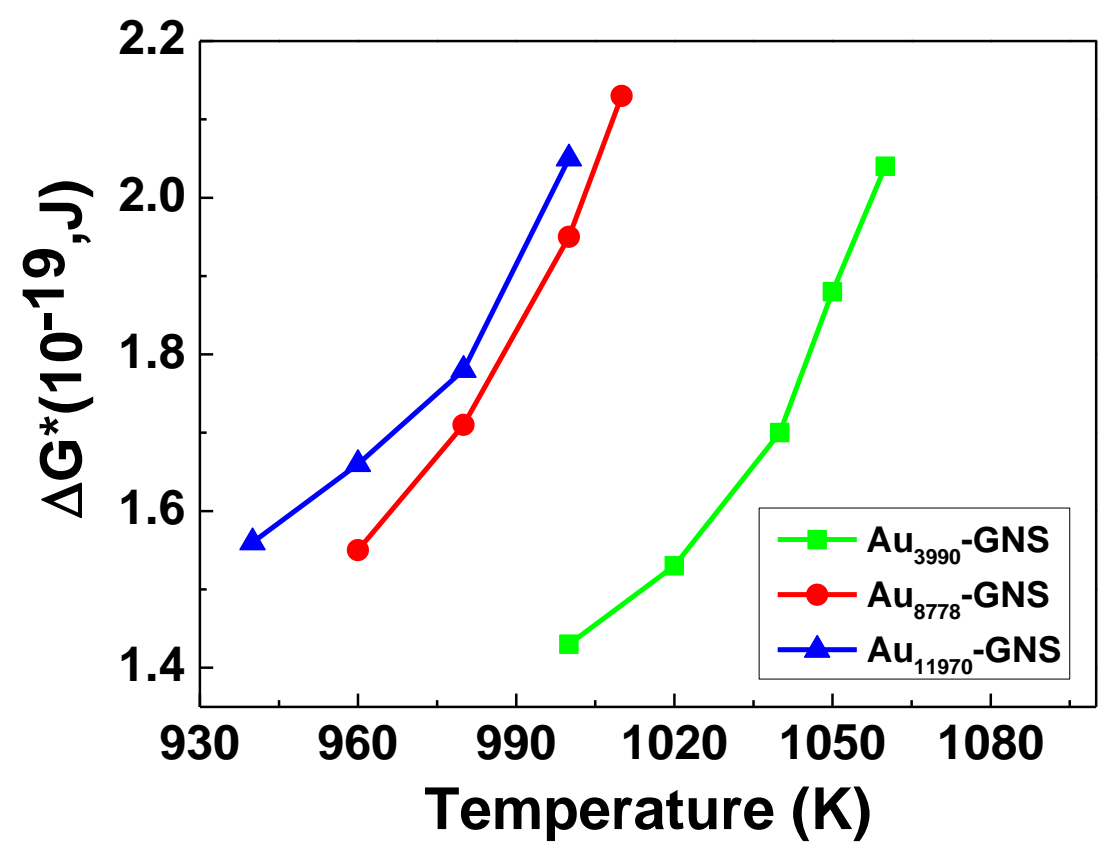

Fig. 7. The relationship between nucleation barriers and temperature for $\mathrm{Au}_{3990},-\mathrm{GNS}, \mathrm{Au}_{8778^{-}} \mathrm{GNS}$, and $\mathrm{Au}_{11970^{-}} \mathrm{GNS}$. 


\section{Table 1}

The thermodynamic data got from MD simulations.

\begin{tabular}{lccl}
\hline System $^{\mathrm{a}}$ & $\Delta \mathrm{C}_{\mathrm{p}}\left(\mathrm{C}_{\mathrm{p}}(\right.$ solid $)-\mathrm{C}_{\mathrm{p}}($ liquid $\left.)\right)\left(\mathrm{J} \mathrm{K}^{-1} \mathrm{~mol}^{-1}\right)$ & $\mathrm{T}_{\mathrm{m}}(\mathrm{K})$ & $\Delta \mathrm{H}_{\text {fus }}\left(\mathrm{kJ} \mathrm{mol}^{-1}\right)$ \\
\hline $\mathrm{Au}_{3990}$-GNS & 6.53 & 1148 & 8.77 \\
$\mathrm{Au}_{8778}$-GNS & 5.40 & 1075 & 8.20 \\
$\mathrm{Au}_{11970}-\mathrm{GNS}$ & 5.31 & 1064 & 7.95 \\
$\mathrm{Au}_{17157}-\mathrm{GNS}$ & 4.77 & 1050 & 7.84 \\
Free bulk Au(exp) & & $1336^{\mathrm{b}}$ & $12.4^{\mathrm{c}}$ \\
\hline
\end{tabular}

a $\mathrm{Au}_{\mathrm{N}-\mathrm{GNS}}$ accounts for a gold nanoparticle is confined between two-layer GNSs.

b I. Barin , O. Knacke, Thermochemical Properties of Inorganic Substances, Springer-Verlag :Berlin, 1973.

c R. C. Weast (9Ed.) CRC Handbook of Chemistry and Physics, $63^{\text {rd }}$ edn., CRC Press, Boca Raton, FL, 1982. 
Table 2

Nucleation rates $\left(\times 10^{-35}, \mathrm{~m}^{-3} \mathrm{~s}^{-1}\right)$ and solid-liquid interfacial free energies $\left(\mathrm{mJ} \mathrm{m}^{-2}\right)$ for Au nanoparticles confined between two-layer GNSs.

\begin{tabular}{lrll}
\hline System & $\mathrm{T}(\mathrm{K})$ & $\mathrm{J}$ & $\sigma_{\mathrm{sl}}{ }^{\mathrm{a}}$ \\
\hline $\mathrm{Au}_{3990}$-GNS & 1000 & $11.77(25)$ & 101.3 \\
& 1020 & $17.47(15)$ & 103.0 \\
& 1040 & $2.82(5)$ & 104.7 \\
& 1050 & $0.94(2)$ & 105.5 \\
$\mathrm{Au}_{8778}$-GNS & 1060 & $0.37(3)$ & 106.0 \\
& 960 & $4.15(6)$ & 98.4 \\
& 980 & $1.61(3)$ & 99.2 \\
$\mathrm{Au}_{11970}-\mathrm{GNS}$ & 1000 & $0.37(3)$ & 101.2 \\
& 1010 & $0.12(2)$ & 101.9 \\
& 940 & $4.05(2)$ & 94.6 \\
& 960 & $2.39(4)$ & 96.0 \\
& 980 & $1.32(5)$ & 97.4 \\
& 1000 & $0.25(1)$ & 98.8 \\
\hline
\end{tabular}

${ }^{\mathrm{a}} \sigma_{\mathrm{sl}}$ are obtained from Turnbull relation. 


\section{Highlights}

(1) The confined gold nanoparticles have face center cubic structure.

(2) The confined gold nanoparticles freeze start from the outmost layers.

(3) The freezing transition mechanism of a confined gold cluster is revealed.

(4) The two-layer-GNS support with smaller hole diameter can stabilize smaller gold nanoparticles.

(5) Some important kinetic parameters of confined gold nanoparticles are estimated. 


\section{Graphical abstract \\ Graphical abstract}
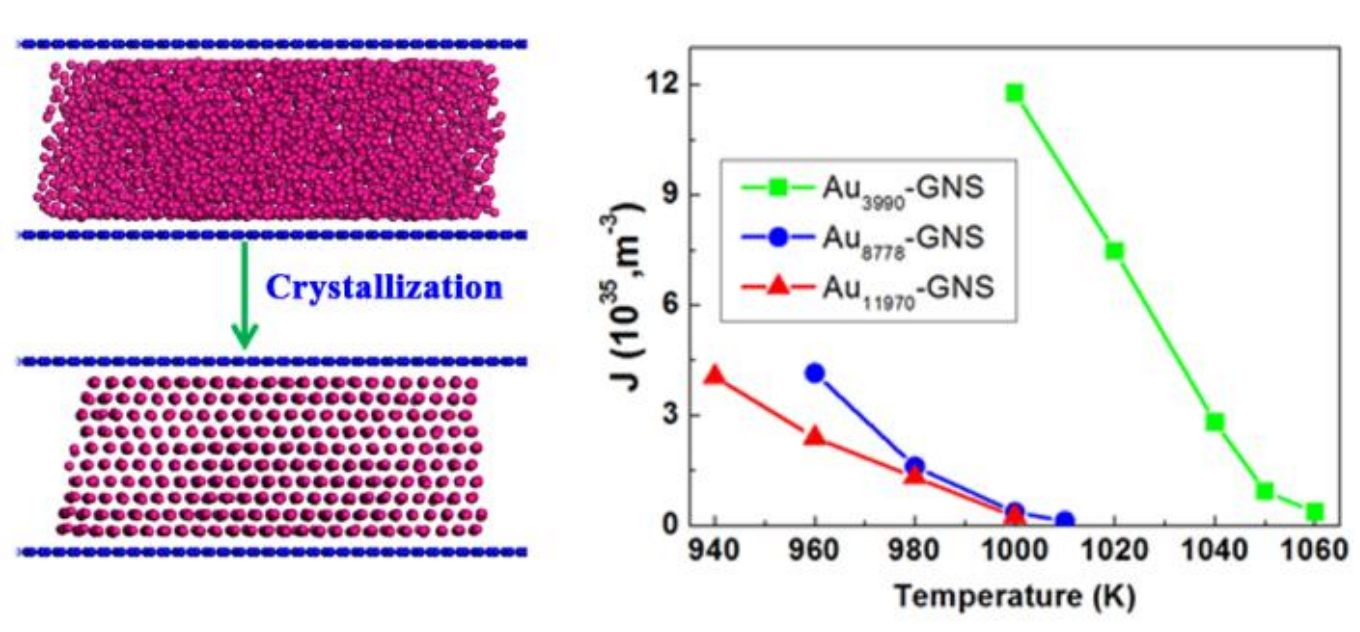